Check for updates

Cite this: RSC Adv., 2017, 7, 23982

Received 14th February 2017

Accepted 24th April 2017

DOI: $10.1039 / \mathrm{c} 7 \mathrm{ra01865g}$

rsc.li/rsc-advances

\section{Incorporation of well-dispersed calcium phosphate nanoparticles into PLGA electrospun nanofibers to enhance the osteogenic induction potential $\uparrow$}

\author{
Dandan Wang, (D) ab Liuyang Xuan, ${ }^{\text {ab }}$ Huixiang Zhong, (D) ab Yihong Gong, ${ }^{\text {ab }}$ \\ Xuetao Shi, ${ }^{c}$ Feng Ye, ${ }^{d}$ Yan Li (D) *ab and Qing Jiang ${ }^{\text {ab }}$
}

Poly(lactide-co-glycolide) (PLGA) electrospun composite nanofibers were fabricated to mimic bone extra cellular matrix for applications in bone tissue engineering. Poly(acrylic acid) modified $\mathrm{Zn}$-doped calcium phosphate (PAA-CaP/Zn) nanoparticles were first synthesized. PAA was selected because of the strong bonding ability with calcium and $\mathrm{Zn}$ was incorporated to enhance the bioactivity. PAA-CaP/Zn nanoparticles were well dispersed with a hydrodynamic size of $184 \mathrm{~nm}$; the presence of $\mathrm{Zn}$ was confirmed and the composition was similar to hydroxyapatite. These nanoparticles were non-cytotoxic against adipose derived stem cells from rats ( $\mathrm{ADSC}$ ) at $0.1 \mathrm{mg} \mathrm{m}^{-1}$ based on an extraction assay and direct contact method. The nanoparticles were further entrapped into PLGA nanofibers. All electrospun membranes were composed of smooth fibers and for composite membranes, nanoparticles were homogeneously distributed in the fiber matrix. Furthermore, the tensile strength was increased with compromised elasticity, while the degradation was retarded and the biomineralization property was enhanced due to the presence of nanoparticles. rADSC proliferated very well on composite membranes and the one with 5\% nanoparticles (P-5) demonstrated the highest osteogenic induction effect as significantly more calcium was deposited after osteogenic induction for 21 days. Sample P-5 was the most suitable for applications in bone tissue engineering.

\section{Introduction}

Tissue engineering combines the developments in material science and cell biology to repair defects which are congenital or have resulted from accidents/diseases. It is an important technology to minimize patients' pain. ${ }^{\mathbf{1}}$ Bone tissue engineering is one branch, mainly focused on repairing bone defects. For such purposes, scaffold materials are indispensible, which should be biocompatible, bioactive and biodegradable. The degradation by-products of ideal scaffolds need to also be biocompatible so

${ }^{a}$ Department of Biomedical Engineering, Guangdong Provincial Key Laboratory of Sensor Technology and Biomedical Instrument, School of Engineering, Sun Yat-sen University, Guangzhou, Guangdong, P. R. China. E-mail: liyan99@mail.sysu.edu.cn; Fax: +86-20-39387890; Tel: +86-20-39387890

${ }^{b}$ Guangdong Provincial Engineering and Technology Center of Advanced and Portable Medical Devices, Sun Yat-sen University, Guangzhou, Guangdong, P. R. China

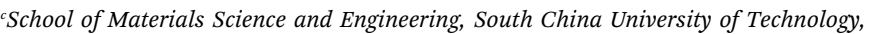
Guangzhou, P. R. China

${ }^{d}$ Key Laboratory of Molecular Biology for Infectious Diseases, Ministry of Education of China, The Second Affiliated Hospital, Chongqing Medical University, Chongqing, P. R. China

$\dagger$ Electronic supplementary information (ESI) available: SEM image and fiber diameter distribution of P-8 which was fabricated at weight ratio of (PAA-CaP/Zn)/PLGA as $8 \%$ and following the same parameters as that for other PLGA electrospun membranes. See DOI: 10.1039/c7ra01865g as not to trigger undesired responses such as immune rejection or inflammation, which minimize the influence on the microenvironment of implants and furthermore lead to patients' pain. $^{2}$ For such applications, there are three kinds of scaffold material: organic, inorganic and organic/inorganic composite materials. Among them, poly(lactide-co-glycolide) (PLGA), which is biocompatible with a controllable degradation rate re $^{3,4}$ and its degradation by-products are also biocompatible, has been widely used. A PLGA scaffold has been applied to repair rat scull defects; on scaffolds, cells proliferated very well and the repair outcome was good. ${ }^{5}$

PLGA scaffolds were prepared with various methods, such as particle leaching, phase separation, lyophilization, selfassembly and electrospinning. Among these techniques, electrospinning has attracted lots of attentions because of its reproducibility, large specific area of the electrospun membrane, maneuverability of fiber diameter (could be in nano-meter or micro-meter range) and flexibility of surface morphology. ${ }^{\mathbf{1 , 6}}$ The scaffold fabricated with electrospinning not only mimicked the structure of extra cellular matrix (ECM), but also offered sufficient space for cell proliferation and nutrient transportation due to its porous structure, which further improved the biocompatibility of scaffolds. ${ }^{7,8}$

To further mimic the composition of bone ECM, inorganic materials such as hydroxyapatite (HAp) was embedded into PLGA 
electrospun fibers to form composite membranes. ${ }^{9-13}$ The compositions and properties of such membranes were intensively studied. Different amounts of HAp nanoparticles (1, 5, 10 and 20 wt\%) were encapsulated into PLGA fibers. With the increase of HAp amount, the average fiber diameter increased from $300 \mathrm{~nm}$ (pure PLGA) to $700 \mathrm{~nm}$ (with 20\% HAp) while the glass transition temperature $\left(T_{\mathrm{g}}\right)$ also increased. Regarding the dynamic mechanical behavior, HAp nanoparticles acted as reinforcements at lower concentrations (1\% and 5\%), but acted as defects at higher concentrations (10\% and 20\%). HAp is hydrophilic, which influenced the uptake of PBS and the mass loss of composite membranes. ${ }^{11}$ On PLGA with 5 wt\% HAp electrospun composite membrane, MC3T3-E1 cells showed a more spreading morphology and significant higher alkaline phosphatase (ALP) secretion. ${ }^{13}$ The effect of HAp morphology on cell behaviors was investigated. ${ }^{9}$ Needle-like or spherical HAp nanoparticles were entrapped in PLGA nanofibers. In vitro tests demonstrated that a higher cell (MC3T3-E1) proliferation and enhanced osteogenesis was observed on composite membranes with needle-like HAp. ${ }^{9}$ The size of HAp particles also played an important role in cell behaviors. ${ }^{10}$ Poly(L-lactide) (PLLA) composite scaffolds were prepared by incorporating needle-shaped nano- or micro-sized HAp particles into PLLA nanofibers using electrospinning, which had higher relative cell (rat osteosarcoma ROS17/2.8 cells) viabilities and ALP activities than the pure PLLA scaffold, especially the scaffold with micro-sized HAp particles. ${ }^{10}$ The presence of HAp nanoparticles in PLGA composite electrospun scaffolds significantly enhanced the formation of bonelike apatites and also the proliferation of primary human osteoblast (HOB). Additionally, such composite scaffold promoted the response of HOB better than MC 3T3 E1. ${ }^{12}$

Besides incorporating HAp, composite membranes of more complex compositions and structures were fabricated. ${ }^{\mathbf{1 4}, 15}$ The incorporation of gelatin/HAp into PLGA electrospun nanofibers decreased static contact angle, increased swelling ratio, facilitated adhesion, migration and proliferation of bone cells (MC3T3-E1), as well as the osteogenic differentiation of progenitor cells (mesenchymal stem cells, MSC) based on the results of osteogenic genes expression. ${ }^{\mathbf{1 4}} \mathrm{A}$ double membrane consisting of a PLGA layer and an amorphous calcium phosphate/collagen/PLGA composite layer were synthesized using electrospinning, which was used for treatment of bone defects with complex geometries as wound dressing material. ${ }^{15}$ Biomineralization on such anisotropic membrane was preferably on the composite layer side. Differentiation of human MSC (hMSC) into the osteogenic lineage was better than that in a $2 \mathrm{D}$ control. ${ }^{15}$ All these composite membranes demonstrated better bioactivity. However, none of these reported structures addressed the dispersity of HAp nanoparticles in polymeric matrix, where HAp nanoparticles were not surface modified. Thus when a higher amount of HAp was encapsulated ( $>5 \%)$, agglomeration of HAp was present, the morphology of nanofibers was also affected ${ }^{\mathbf{1 1}, 13}$ and mechanical properties were compromised. Additionally, in these structures, HAp only contained $\mathrm{Ca}$ as the cation ion, which was different from the in vivo composition. Hence for better performance in bone tissue engineering, the composite membrane with improved structure needed to be elaborately designed.

Besides calcium, other metal ions such as $\mathrm{Mg}, \mathrm{Mn}$ and $\mathrm{Zn}^{\mathbf{1 6}}$ are present in bone minerals. Even these elements are in small amount, they are fundamental to bone metabolism. $\mathrm{Zn}$ is an essential element for human health and also important for bone metabolism. Zn was reported to increase the proliferation and differentiation of osteoblast cells, ${ }^{17}$ and also suppressed the activation of osteoclast cells. ${ }^{18}$ Thus in this paper $\mathrm{Zn}$ was selected to fabricate $\mathrm{Zn}$ doped HAp-like calcium phosphate (CaP/Zn) nanoparticles for better bioactivity.

To improve the dispersity, organic additives, such as polyethylene glycol (PEG), ${ }^{19}$ cetyl trimethylammonium bromide $(\mathrm{CTAB})^{20}$ and citric acid ${ }^{19}$ were applied to fabricate HAp nanoparticles. However, these additives either did not bind HAp strongly or were surfactants which may not be suitable for scaffolds applications because of the cytotoxicity. Thus poly(acrylic acid) (PAA) with lots carboxyl groups which can bind HAp strongly and flexible long molecular chains which may facilitate the distribution of nanoparticles in polymeric matrix, was selected as the organic additive for the fabrication of HAp-like calcium phosphate nanoparticles.

In this paper, well dispersed $\mathrm{Zn}$ doped HAp-like calcium phosphate nanoparticles were fabricated in the presence of PAA (PAA-CaP/Zn), which were further embedded into PLGA nanofibers using electrospinning technique. To our best knowledge, this composite structure was first developed. The structure and properties of PAA-HAp/Zn nanoparticles and PLGA electrospun composite membranes were intensively studied. The electrospun composite membrane was designed for bone tissue engineering applications. As few studies have investigated the degradation behavior and also the osteogenic differentiation potential of adipose-derived stem cells (ADSC) on such membranes, its degradation behavior was monitored, the proliferation and osteogenic differentiation potential of ADSC from rats (rADSC) were also investigated.

\section{Materials and methods}

\subsection{Materials}

Acetone (99.5\%), calcium chloride $\left(\mathrm{CaCl}_{2}\right)$, dimethyl formamide (DMF, 99.5\%), ethanol (95\%), sodium hydrogen phosphate $\left(\mathrm{Na}_{2} \mathrm{HPO}_{4} \cdot 12 \mathrm{H}_{2} \mathrm{O}\right)$ and zinc chloride $\left(\mathrm{ZnCl}_{2}\right)$ were bought from Guangzhou Chemical Reagent Co. (Guangzhou, Guangdong, China). PAA $\left(M_{\mathrm{w}} 5000\right)$ was from Shanghai Ryon Biological Technology CO., Ltd (Shanghai, China) and PLGA (lactide/ glycolide molar ratio 50/50, $\left.M_{\mathrm{w}}=70000-80000\right)$ was from Jinan Daigang Biological Technology Co. (Jinan, Shandong, China). Dulbecco's Modified Eagle Media: Nutrient Mixture F-12 (DMEM/F12) and PS (penicillin/streptomycin antibiotics) were purchased from HyClone (Logan, UT, USA). Fetal bovine serum (FBS) and trypsin-EDTA were purchased from Gibco (Carlsbad, CA, USA). Alamar Blue was from Sigma-Aldrich (St, Louis, MO, USA) and a BCIP/NBT ALP Color Development Kit was from Beyotime (Haimen, Jiangsu, China). All chemicals were used without further purification unless otherwise stated. Deionized (DI) ultrapure water was used throughout the experiment. 


\subsection{PAA modified Zn doped calcium phosphate (PAA-CaP/} Zn) nanoparticles

2.2.1 Fabrication of PAA-CaP/Zn nanoparticles. PAA was first dissolved in DI water at $5 \mathrm{mg} \mathrm{ml}{ }^{-1}$. $\mathrm{CaCl}_{2}(0.1055 \mathrm{~g})$ and $\mathrm{ZnCl}_{2}(0.0068 \mathrm{~g})$ were both dissolved in $10 \mathrm{ml}$ of PAA solution to achieve the molar ratio of $\mathrm{Zn} / \mathrm{Ca}$ at 5/95 in $\mathrm{HAp} ; \mathrm{Na}_{2} \mathrm{HPO}_{4} \cdot 12 \mathrm{H}_{2} \mathrm{O}$ $(0.2148 \mathrm{~g})$ was dissolved in $100 \mathrm{ml}$ of PAA solution. The $\mathrm{CaCl}_{2}$ solution was slowly added at $0.5 \mathrm{ml} \mathrm{min}{ }^{-1}$ into $\mathrm{Na}_{2} \mathrm{HPO}_{4} \cdot 12 \mathrm{H}_{2} \mathrm{O}$ under stirring at $400 \mathrm{rpm}$. The reaction was processed at room temperature and the $\mathrm{pH}$ value of mixture was maintained at $\sim 10$ using $\mathrm{NaOH}$ solution $(1 \mathrm{M})$. After reacted for $2 \mathrm{~h}$, the product was collected by centrifugation at $3000 \mathrm{rpm}$, rinsed three times with DI water and freeze-dried before storage.

2.2.2 Characterization of PAA-CaP/Zn nanoparticles. The crystal phase of product was investigated using X-ray diffraction (XRD, D/Max-IIIA, Rigaku, Japan) with $\mathrm{Cu} \mathrm{K} \alpha$ radiation $(\lambda=$ $1.78897 \AA$ ) at a voltage of $40 \mathrm{kV}$ and current of $40 \mathrm{~mA}$. The data was collected in the range of $20-60^{\circ}$ at $0.05^{\circ} \mathrm{s}^{-1}$. The functional groups in samples were studied using a Fourier transform infrared (FTIR) spectrophotometer (VERTEX 70, Bruker Optics, Germany) with $\mathrm{KBr}$ pellet technique in the range of 4000-400 $\mathrm{cm}^{-1}$ with a resolution of $4 \mathrm{~cm}^{-1}$. The morphology of sample was observed using a scanning electron microscopy (SEM, JSM5600, JEOL, Tokyo, Japan) after samples were sputter coated with gold. The hydrodynamic size and zeta potential of nanoparticles in mother suspension ( $\mathrm{pH} 10)$ when reaction was stopped were quantified using a Zetasizer (Nano-ZS90, Malvern, $\mathrm{UK}$ ). The amount of $\mathrm{Zn}$ and $\mathrm{Ca} / \mathrm{P}$ atomic ratio of samples was quantified using Inductively Coupled Plasma-Atomic Emission Spectrometry (ICP-AES, IRIS (HR), TJA, USA) after sample was dissolved in nitric acid.

2.2.3 Cytotoxicity evaluation of PAA-CaP/Zn nanoparticles. rADSC were used to evaluate the in vitro cytotoxicity of PAA-CaP/ $\mathrm{Zn}$ nanoparticles and were isolated from SD rats. All isolation procedures were referred to a publication paper, ${ }^{21}$ which followed the guideline of Institutional Animal Care and Use Committee and were also approved by the Animal Ethical and Welfare Committee (AEWC) of Sun Yat-sen University. Cells were cultured in proliferation medium which was DMEM/F12 supplemented with $10 \%$ FBS and 1\% PS.

The cytotoxicity of samples was investigated using extraction assay and direct contact assay. rADSC at passage 3 (P3) were seeded into 96-well plates at 5000 cell per $\mathrm{cm}^{2}$. Samples in powder were sterilized by soaking in $75 \%$ ethanol for 3-4 $\mathrm{h}$ and rinsed with PBS three times. For extraction assay, the sterilized powder was first suspended in DMEM/F12 medium at $200 \mathrm{mg}$ $\mathrm{ml}^{-1}$ for $24 \mathrm{~h}$ in an incubator at $37{ }^{\circ} \mathrm{C} ; ;^{22,23}$ the supernatant was then collected by centrifugation, further filtration through 0.22 $\mu \mathrm{m}$ filter and diluted twice with fresh medium. For direct contact assay, the sterilized powder was suspended in medium at $0.1 \mathrm{mg} \mathrm{ml} \mathrm{m}^{-1}$. When cells reached $80 \%$ confluence, the medium was exchanged with extraction medium or sample/ medium suspension. Cells cultured in normal medium were negative controls while in medium containing $0.1 \%$ Triton $\mathrm{X}$ 100 were positive controls. On day 1 and 3, Alamar Blue assay was conducted to quantify cell viabilities.

\subsection{PLGA electrospun composite membranes}

2.3.1 Fabrication of PLGA electrospun composite membranes. The fabrication process was shown in Scheme 1. In detail, PLGA was dissolved in DMF. PAA-CaP/Zn at $0,1 \%$ and 5\% of PLGA was weighted and suspended in acetone by ultrasonic dispersion. The volumetric ratio of acetone/DMF was kept at 3/1. The PAA-CaP/Zn suspension was added into PLGA solution under stirring (the final PLGA concentration was $20 \mathrm{wt} \%$ ) and the suspension was further stirred until nanoparticles were well suspended (the suspension was homogenous and even placed for $\sim 2$ days without stirring, there was no precipitate settled). The suspensions were electrospun to be sample P (pure PLGA), P-1 (CaP/PLGA 1\%) and P-5 (CaP/PLGA 5\%). The composite membranes were collected on an aluminum foil. For in vitro degradation, biomineralization and cell studies, samples P, P-1 and P-5 were adhered onto cover slips (diameter of $\sim 14 \mathrm{~mm}$ ) which were suitable to be placed in 24 -well plates. The detailed set-up parameters for electrospinning were as following: voltage $14 \mathrm{kV}$, injection rate $1 \mathrm{ml} \mathrm{h}^{-1}$, collection distance $20 \mathrm{~cm}$ (the distance between the needle tip and aluminum foil) and injection needle $21 \#$ ( $\varphi=0.22 \mathrm{~mm}$, length $15 \mathrm{~cm})$.

2.3.2 Characterization of PLGA electrospun composite membranes. The morphology of membranes was observed using a SEM after sputter coated with gold. The diameters of fibers were measured using software Image J. The distribution of $\mathrm{PAA}-\mathrm{CaP} / \mathrm{Zn}$ nanoparticles in electrospun fiber was investigated using TEM (JEM-1400, JEOL, Tokyo, Japan). The mechanical properties were evaluated using a Microcomputer control electronic universal testing machine (CMT4104, MTS, USA). Samples were cut into rectangles at $70 \times 15 \mathrm{~mm}$. The distance of clamp was set at $50 \mathrm{~mm}$ and the strain rate was 10 $\mathrm{mm} \min ^{-1}$ until sample was ruptured. The glass transition temperature $\left(T_{\mathrm{g}}\right)$ was quantified using a Differential Scanning Calorimeter (DSC, Q20, TA Instruments, USA). Membrane of $\sim 5 \mathrm{mg}$ was placed in an aluminum crucible. Under nitrogen gas the sample was heated from $0{ }^{\circ} \mathrm{C}$ to $100{ }^{\circ} \mathrm{C}$ at a rate of $10{ }^{\circ} \mathrm{C}$ $\min ^{-1}$. The $T_{\mathrm{g}}$ was defined as the temperature at which the slope of DSC thermographs was the largest during glass transition.

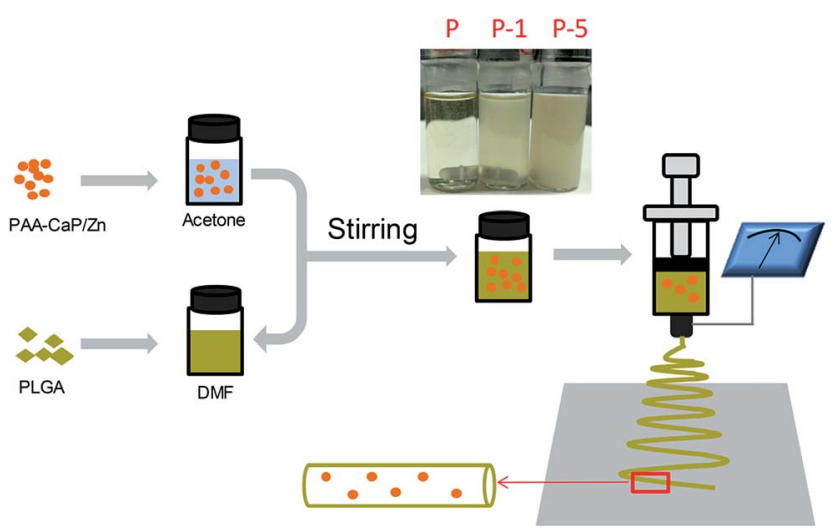

Scheme 1 Fabrication of PLGA composite membranes with welldispersed PAA-CaP/Zn nanoparticles using electrospinning technique. 
2.3.3 In vitro degradation studies of PLGA electrospun composite membranes. Electrospun membranes P, P-1 and P-5 were placed in 24-well plates. $1 \mathrm{ml}$ of PBS was added to each well and incubated in a water bath at $37{ }^{\circ} \mathrm{C}$. PBS was replenished every 2 days. After degraded for 7, 14, 21 and 28 days, membranes were taken out, rinsed with DI water and freezedried. The surface morphology was observed under SEM. For each sample at each time points, two replicates were investigated.

2.3.4 In vitro biomineralization evaluations of PLGA electrospun composite membranes. Samples P, P-1 and P-5 were placed in 24-well plates. After sterilized by soaking in $75 \%$ alcohol for $\sim 4 \mathrm{~h}$ and dried in a laminar flow hood, $1 \mathrm{ml}$ simulated body fluid (SBF) was added to each well. SBF was prepared according to a reported protocol. ${ }^{24}$ These membranes were incubated at a $37^{\circ} \mathrm{C}$ water bath and SBF was exchanged every 2 days. On day 7 and 14, samples were taken out, rinsed with DI water and dried using lyophilization. The surface morphology of these membranes was observed under SEM, and the elements of deposits were characterized using energy dispersive spectroscopy (EDS).

2.3.5 Proliferation of rADSC on PLGA electrospun composite membranes. PLGA electrospun membranes were placed in 24-well plates. Before cell seeding, these membranes were sterilized by soaking in $75 \%$ ethanol for $\sim 4 \mathrm{~h}$ and rinsed with PBS five times to remove remaining ethanol. The P3 rADSC were seeded onto these membranes at 5000 cells per $\mathrm{cm}^{2}$ and cultured in proliferation medium. Cell number was monitored using Alamar Blue assay following manufacturers' descriptions. In brief, the stock solution was first prepared in PBS at $0.1 \mathrm{mg}$ $\mathrm{ml}^{-1}$ and then added into 24 -well plates at a dilution ratio of 1 / 10 with the proliferation medium. Reaction was allowed to proceed for $4 \mathrm{~h}$ in dark and the supernatant was transferred to a 96-well plate for absorbance reading using a microplate reader (BioTek Synergy4, USA). The cell number was represented by the difference of absorbance values at $570 \mathrm{~nm}$ and $600 \mathrm{~nm}$. After cell growing on membranes for 7 days, cell morphology was observed under SEM after fixed with $2.5 \%$ glutaraldehyde and lyophilization.

2.3.6 Osteogenic differentiation of rADSC on PLGA composite membranes. rADSC were seeded on sterilized membranes at 20000 cells per $\mathrm{cm}^{2}$ in proliferation medium. After $24 \mathrm{~h}$, proliferation medium was changed to osteogenic medium which was proliferation medium further supplemented with $10 \mathrm{mM} \beta$-glycerophosphate, $0.2 \mathrm{mM}$ L-ascorbic acid and $0.1 \mu \mathrm{M}$ dexamethasone. Cells were then cultured for 21 days during which osteogenic medium was exchanged every 2 days. Expression of ALP was qualitatively and quantitatively evaluated using a BCIP/NBT ALP Color Development Kit and $p$ nitrophenylphosphate ( $p \mathrm{NPP})$ assay respectively. On day 21, deposition of calcium minerals was investigated using Alizarin red staining. The amount of stains was quantified using a microplate reader based on the absorbance value at $420 \mathrm{~nm}$ after dissolved with diluted acidic solution, which represented the amount of deposited minerals.

\section{Results}

\subsection{Fabrication and characterization of PAA-CaP/Zn nanoparticles}

In this paper, calcium phosphate nanoparticles were fabricated using wet chemical precipitation method. In order to improve the dispersity, PAA was selected because of its high bonding ability with calcium and to be more bioactive, $\mathrm{Zn}$ was introduced into the reaction system to obtain PAA modified $\mathrm{Zn}$ doped HAp-like calcium phosphate nanoparticles (PAA-CaP/ $\mathrm{Zn)}$. The crystal phase was investigated using XRD. As shown in Fig. 1a, XRD diffraction peaks were very broad and it was difficult to assign the lattice planes. Even so, the positions of these broad peaks agreed well with that for HAp with PDF \#090432. The functional groups were investigated using FTIR spectroscopy (Fig. 1b). For PAA-CaP/Zn, the adsorption band at $1576 \mathrm{~cm}^{-1}$ and $1418 \mathrm{~cm}^{-1}$ were due to $-\mathrm{C}-\mathrm{O}-\mathrm{H}$ and $-\mathrm{C}=\mathrm{O}$ of PAA, where these bands shifted to lower wavenumbers when compared with that of PAA. It was reasonable that in PAA, - $\mathrm{COOH}$ groups were acidic while in $\mathrm{PAA}-\mathrm{CaP} / \mathrm{Zn}$, there were probably ionic interactions between $-\mathrm{COO}^{-}$groups and $\mathrm{Ca} .^{25,26}$ Additionally, there were adsorption bands at $1065 \mathrm{~cm}^{-1}$ and 572 $\mathrm{cm}^{-1}$, which can be assigned to $\mathrm{PO}_{4}{ }^{3-}$ groups in HAp. ${ }^{27,28}$

As shown in Fig. 1c, the formed nanoparticles were in spheroid morphology of $\sim 60 \mathrm{~nm}$. Due to the modification of $\mathrm{PAA}$, these PAA-CaP/Zn nanoparticles were well dispersed in aqueous solution. In mother suspension $(\mathrm{pH} 10)$ when the reaction was stopped, the average size was determined to be $184 \mathrm{~nm}$ based on Zetasizer characterization and the size distribution was narrow. In addition, the zeta potential was measured to be $-14.2 \mathrm{mV}$. The average size measured by Zetasizer was larger than the particle size based on SEM image. There might be two possible reasons. The size determined with Zetasizer was hydrodynamic size. In mother suspension $(\mathrm{pH}$ 10), these surface-bonded PAA molecules may form a layer around these nanoparticles and attract some water molecules. Meanwhile, there might be slight aggregation of nanoparticles. Hence the average size from Zetasizer for $\mathrm{PAA}-\mathrm{CaP} / \mathrm{Zn}$ in suspension was larger.

The presence of $\mathrm{Zn}$ in $\mathrm{PAA}-\mathrm{CaP} / \mathrm{Zn}$ nanoparticles was confirmed with EDS analysis. Besides $\mathrm{Ca}$ and $\mathrm{P}$, a small excitation peak belonging to $\mathrm{Zn}$ was observed (Fig. 1d). Furthermore, the atomic ratio was quantified using ICP after nanoparticles were dissolved in nitric acid. For PAA-CaP/Zn, the $\mathrm{Zn} / \mathrm{Ca}$ atomic ratio was measured to be $5.85 \%$ while $\mathrm{Ca} / \mathrm{P}$ atomic ratio was 1.586 . These two values were both close to the theoretical values $5.26 \%, 1.584$, respectively by assuming that all added $\mathrm{Zn}$ ions had replaced $\mathrm{Ca}$ ions in the structure. All these comparisons showed that $\mathrm{PAA}-\mathrm{CaP} / \mathrm{Zn}$ had the expected composition.

Cytotoxicity of PAA-CaP/Zn nanoparticles was evaluated using extraction assay and direct contact assay. For TCP group (cell cultured in normal proliferation medium), cells proliferated slowly from day 1 to day 3. When compared with TCP group, no matter cells were cultured with nanoparticles extract or suspension, the absorbance values were slightly higher or 

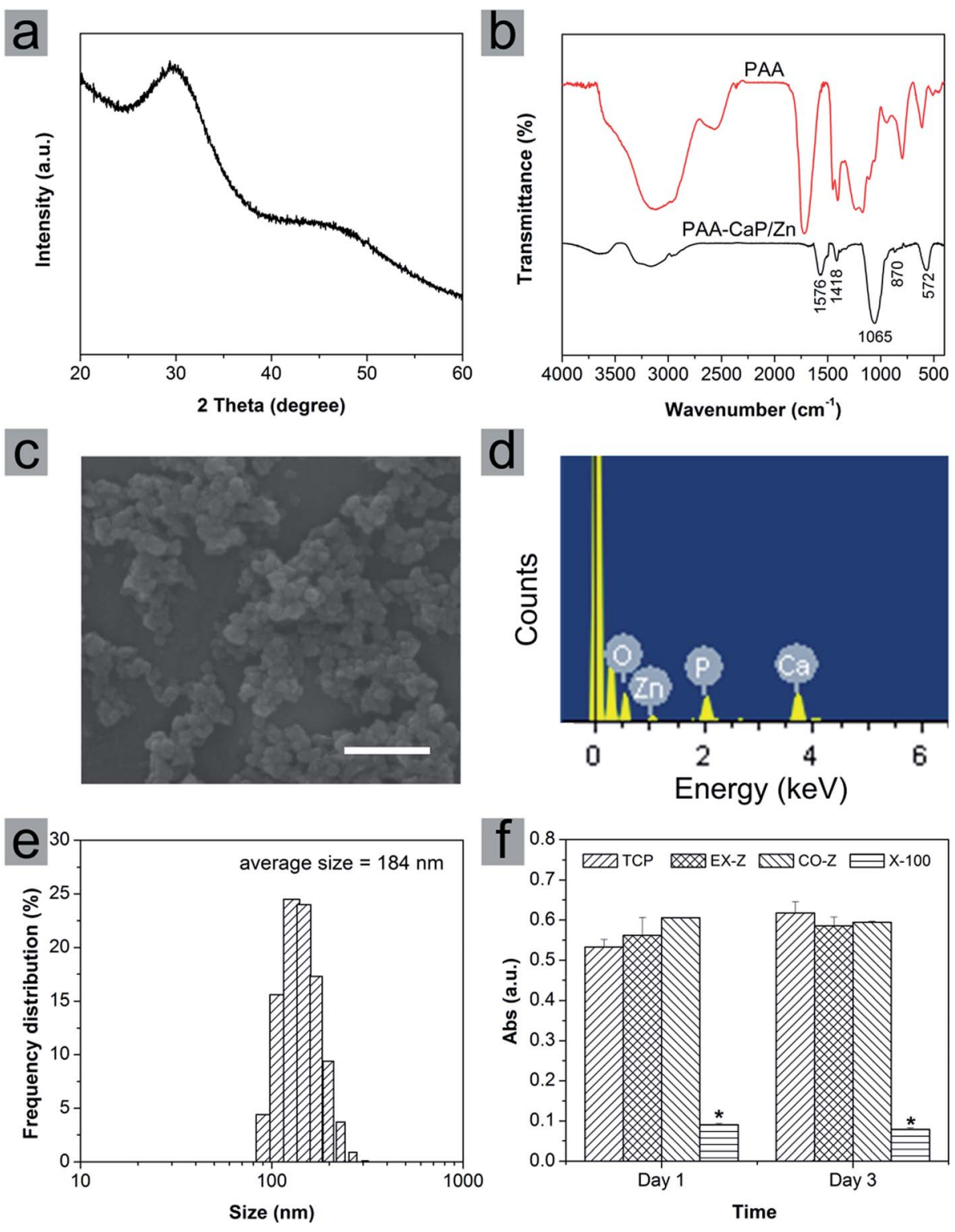

Fig. 1 (a) XRD pattern, (b) FTIR spectrum, (c) SEM image, (d) EDS spectrum, (e) size distribution and (f) cytotoxicity results of PAA-CaP/Zn nanoparticles. Cytotoxicity was investigated using extraction assay (EX-Z group, cells were cultured with extract of PAA-CaP/Zn nanoparticles) and direct contact assay (CO-Z group, cells were cultured in nanoparticles/medium suspension). After cell cultured for 1 and 3 days, Alamar Blue assay was conducted. TCP was the group that cells were cultured in normal proliferation medium and as the negative control; X-100 was the positive control group that cells were cultured with medium containing $0.1 \%$ Triton $\mathrm{X}-100$. Data $=$ mean $\pm \mathrm{SD} ; n=3, *$ representing $p<0.05$ when compared with TCP group on the same day. Scale bar in (c) represents $500 \mathrm{~nm}$.

close to the values for TCP group both on day 1 and 3 . Hence, no toxicity effects on rADSC for PAA-CaP/Zn were observed. As for Triton X-100 group, the absorbance values were significantly smaller, showing that Triton X-100 was toxic against rADSC. These nanoparticles were further entrapped into PLGA electrospun nanofibers.

\subsection{Fabrication and characterization of PLGA electrospun composite membranes}

PLGA electrospun membranes were successfully fabricated following conditions as described in Section 2.3.1. As shown in Fig. 2, all membranes were composed of fibers, where these fibers were smooth, without node-structure and diameters were in the range of 100-1000 $\mathrm{nm}$. The average diameter for P, P-1 and P-5 were $604 \pm 220 \mathrm{~nm}, 415 \pm 128 \mathrm{~nm}$ and $420 \pm$ $142 \mathrm{~nm}$, respectively. Sample P-8 was also fabricated following the same parameters as described in Section 2.3.1 at weight ratio of (PAA-CaP/Zn)/PLGA as $8 \%$. As shown in Fig. S1 (ESI) $\dagger$ the membrane was still fiber-like. However, the fiber surface was no longer as smooth as that for sample P. Although the average fiber diameter was $410 \pm 123 \mathrm{~nm}$ (close to that for P-1, P-5), P-8 was not selected for further studies.

The entrapment of $\mathrm{PAA}-\mathrm{CaP} / \mathrm{Zn}$ nanoparticles slightly decreased the fiber diameter. The decrement of fiber diameter 

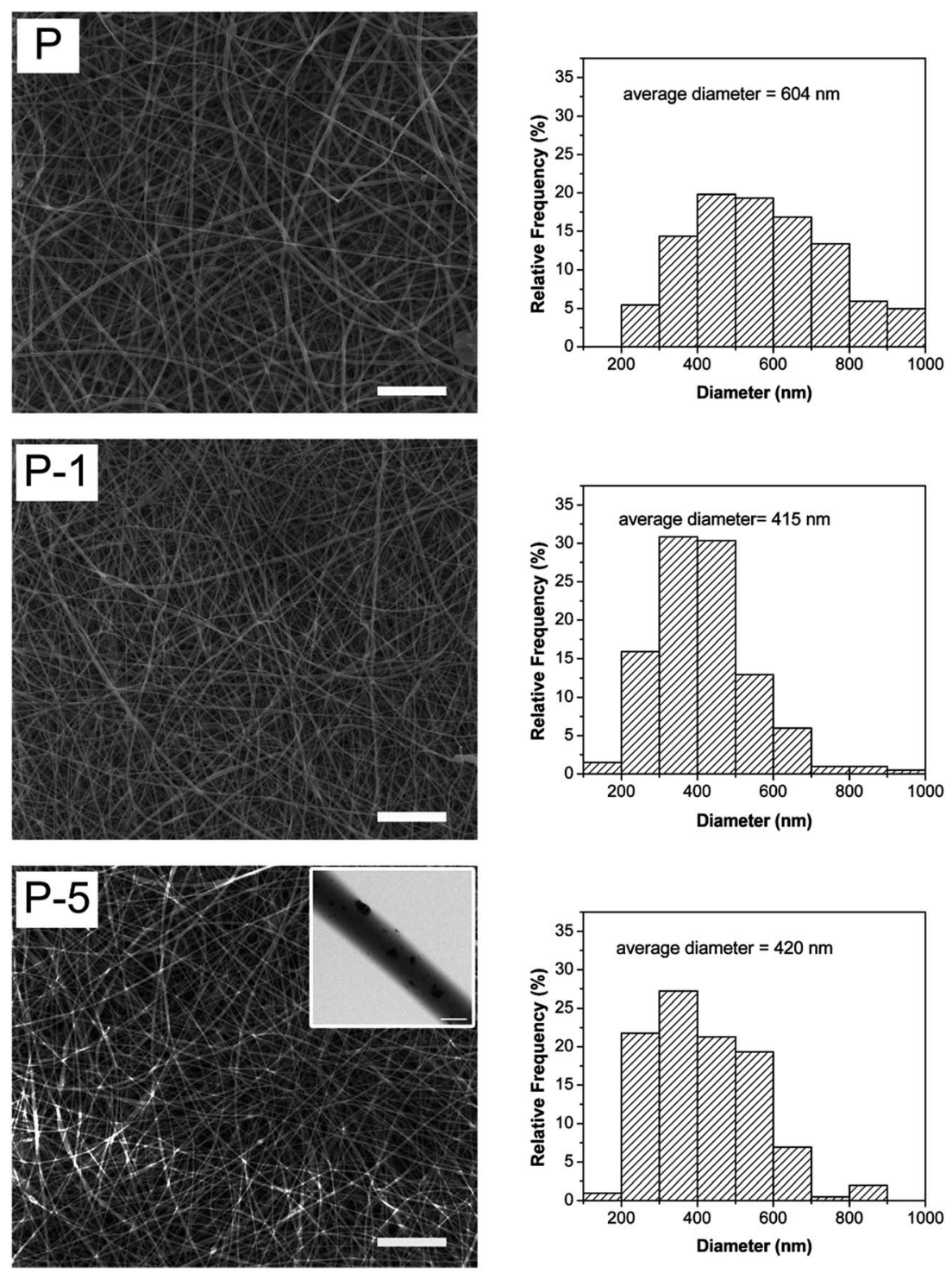

Fig. 2 SEM images and fiber diameter distributions of PLGA electrospun membranes. Scale bars are $20 \mu \mathrm{m}$ (scale bar in the inset is $500 \mathrm{~nm}$ ).

was probably related to PAA molecules on the nanoparticles since PAA-CaP/Zn nanoparticles were found to be well dispersed in the fiber matrix. To verify the speculation, a sample at weight ratio of $\mathrm{PAA} / \mathrm{PLGA}$ as $1 \%$ was fabricated following the same electrospun parameters. For this sample, the average diameter was $310 \pm 114 \mathrm{~nm}$, which was much smaller than that for sample P. Hence when PAA-CaP/Zn nanoparticles were well distributed in polymeric matrix, the fiber diameter was slightly decreased. Using electrospinning method, PLGA composite membranes with similar surface morphology and fiber diameter were obtained.

Mechanical properties of PLGA electrospun membranes (Fig. 3a) were investigated using tensile testing to assess the extensibility of membranes and in turn the interaction between $\mathrm{PAA}-\mathrm{CaP} / \mathrm{Zn}$ nanoparticles and polymeric matrix. For pure PLGA membrane (sample P), the yield stress and strain were 1.9 MPa and $5.83 \%$ while the breaking stress and strain were $2.4 \mathrm{MPa}$ and $93 \%$. After encapsulation of PAA-CaP/Zn nanoparticles, the yield stress was increased to $2.23 \mathrm{MPa}$; the breaking stress increased with the amount of nanoparticles, where for P-1 and $\mathrm{P}-5$, the values were 2.9 MPa and 3.1 MPa respectively. However, the breaking strains of P-1 and P-5 were both less than that for sample P. Elastic modules of these samples were further quantified, which were 2.4 MPa, 3.5 MPa and 3.1 MPa for P, P-1 and $\mathrm{P}-5$, respectively. Based on these comparisons, it was found the presence of nanoparticles increased the tensile strength but compromised elasticity.

The glass transition temperature $\left(T_{\mathrm{g}}\right)$ of PLGA electrospun membranes were quantified using DSC. The DSC thermographs from the first heating cycle were shown in Fig. 3b. For P, P-1 and $\mathrm{P}-5, T_{\mathrm{g}}$ was $53.2{ }^{\circ} \mathrm{C}, 52.8{ }^{\circ} \mathrm{C}$ and $52.4{ }^{\circ} \mathrm{C}$, respectively. The value decreased slightly with the amount of PAA-CaP/Zn increasing. The trend was different from the reported result ${ }^{\mathbf{1 1}}$ that $T_{\mathrm{g}}$ increased with the amount of HAp in PLGA nanofibers. Here the encapsulated nanoparticles were surface modified with PAA and these nanoparticles were well distributed in the fiber 

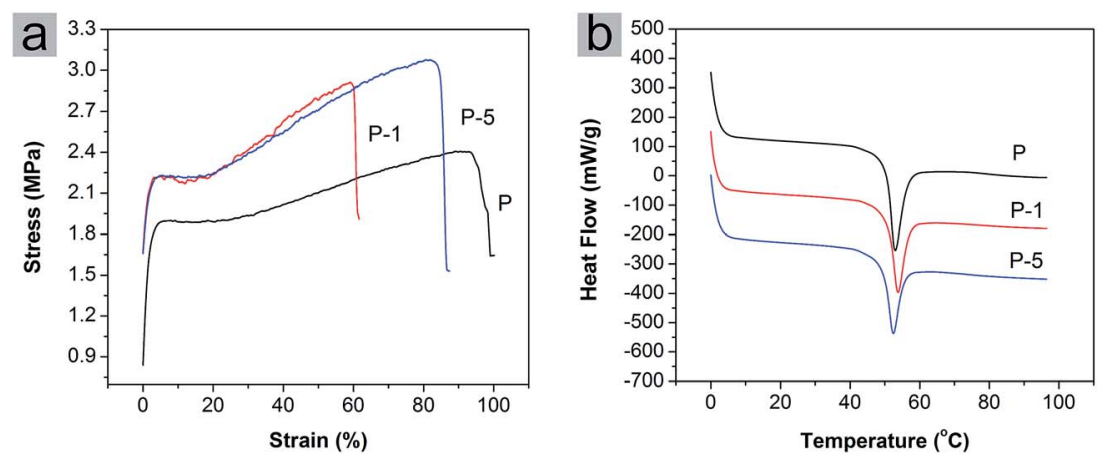

Fig. 3 (a) Stress-strain curves and (b) DSC thermographs of PLGA electrospun membranes P, P-1 and P-5.

matrix. The presence of only PAA molecules in PLGA electrospun membranes decreased the $T_{\mathrm{g}}$ (for PLGA composite membrane with $5 \mathrm{wt} \%$ PAA which was fabricated following the same parameters, the $T_{\mathrm{g}}$ was $52.1^{\circ} \mathrm{C}$ ). All these factors probably resulted in the difference from the reported data.

PLGA is degradable. Here the degradation behavior of PLGA electrospun membranes was evaluated. Through 28 days of degradation, fiber-like morphology of all membranes was maintained (Fig. 4). From day 7 to 21, there was no significant difference among these three samples. However, on day 28, some fiber adhesions were observed on P and P-1, and these two samples appeared to be more flat and dense than sample P-5. As for P-5, fiber-like surface morphology was preserved throughout 28 days of degradation, which might be beneficial for cell proliferation and differentiation.

The in vitro biomineralization properties of PLGA electrospun membranes were evaluated by soaking in SBF. As shown in Fig. 5, on day 7 , there were some crystals deposited on each sample. On sample P, crystals were mainly formed on surface of nanofibers while on surface of P-1 and P-5, the amount of crystals were much

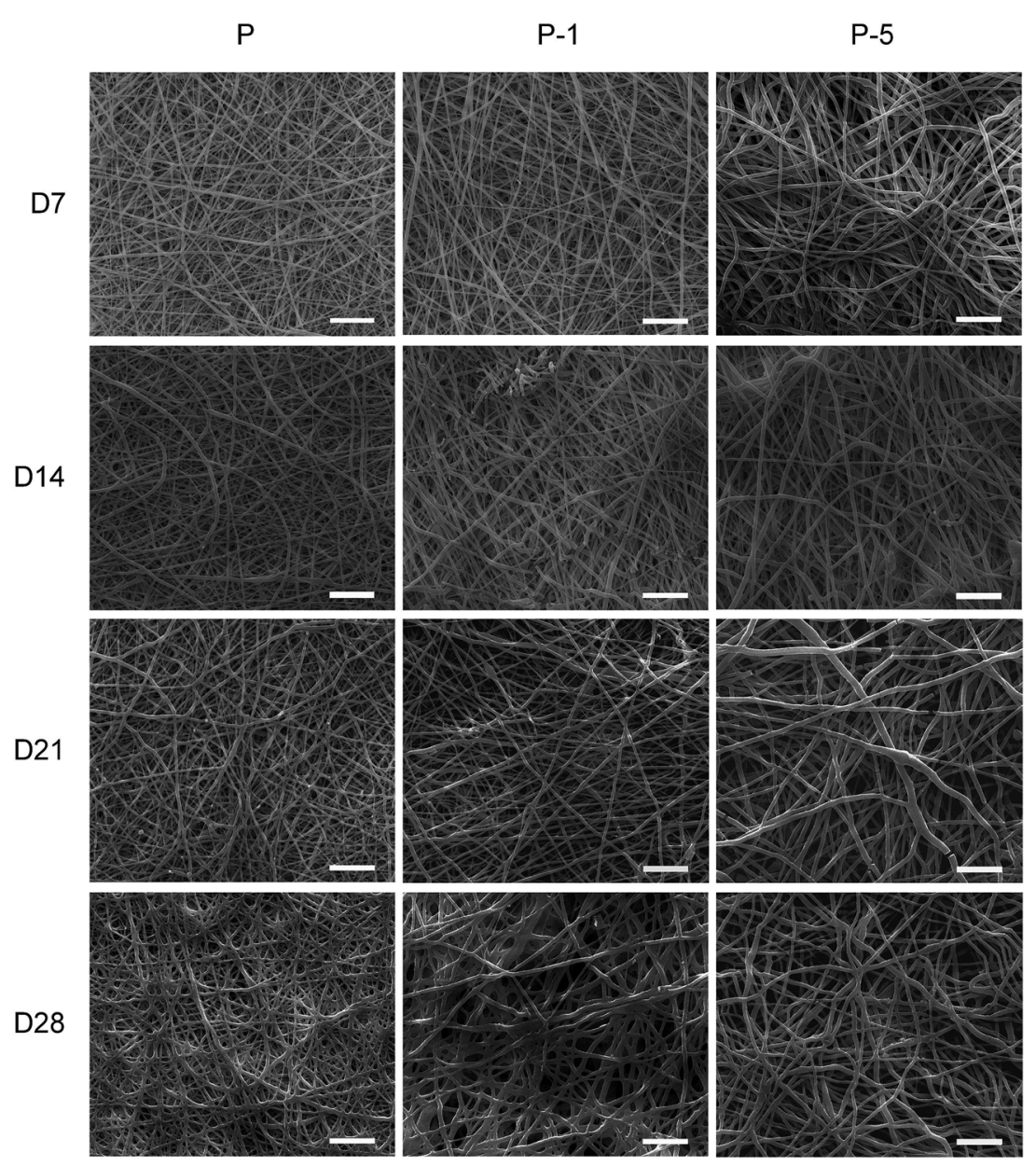

Fig. 4 Surface morphology of samples P, P-1 and P-5 after degraded in PBS for 7, 14, 21 and 28 days. Scale bars are $20 \mu \mathrm{m}$. 
$7 d$
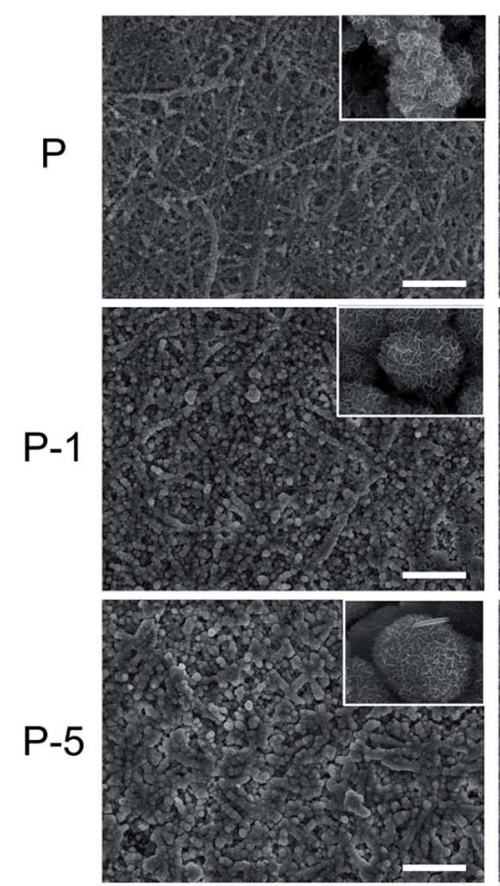

$14 d$
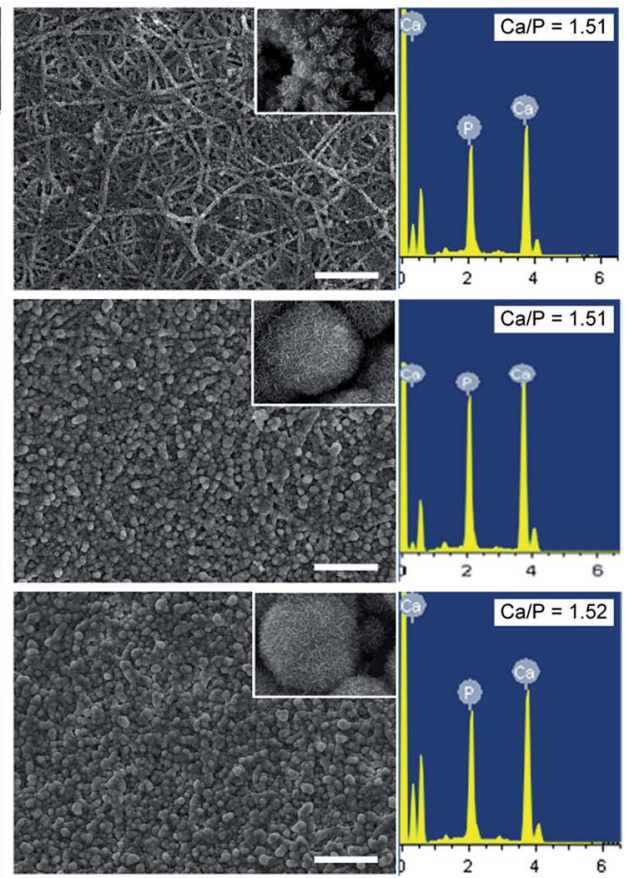

Fig. 5 SEM images of PLGA electrospun membranes after soaked in SBF for 7 and 14 days for in vitro biomineralization evaluation. The insets are enlarged images of deposited crystals. Scale bars are $10 \mu \mathrm{m}$.

more and fiber-like morphology was covered by crystal deposition. When looking at higher magnification (insets in Fig. 5), deposits on sample $\mathrm{P}$ were plate-like and demonstrated the trend to form spherical aggregates; while on surfaces P-1 and P-5, deposits had already formed spherical aggregates, and aggregates on P-5 appeared to be more dense. On day 14, much more deposits were formed on each sample. The morphology of crystals was similar to the reported results. ${ }^{29}$ The elements of deposits were characterized using EDS. As shown in Fig. $5\left(3^{\text {rd }}\right.$ column), both $\mathrm{Ca}$ and $\mathrm{P}$ were detected on all samples and $\mathrm{Ca} / \mathrm{P}$ atomic ratio for sample P-5 was the highest. Combining the morphology results, it can be concluded that the deposited
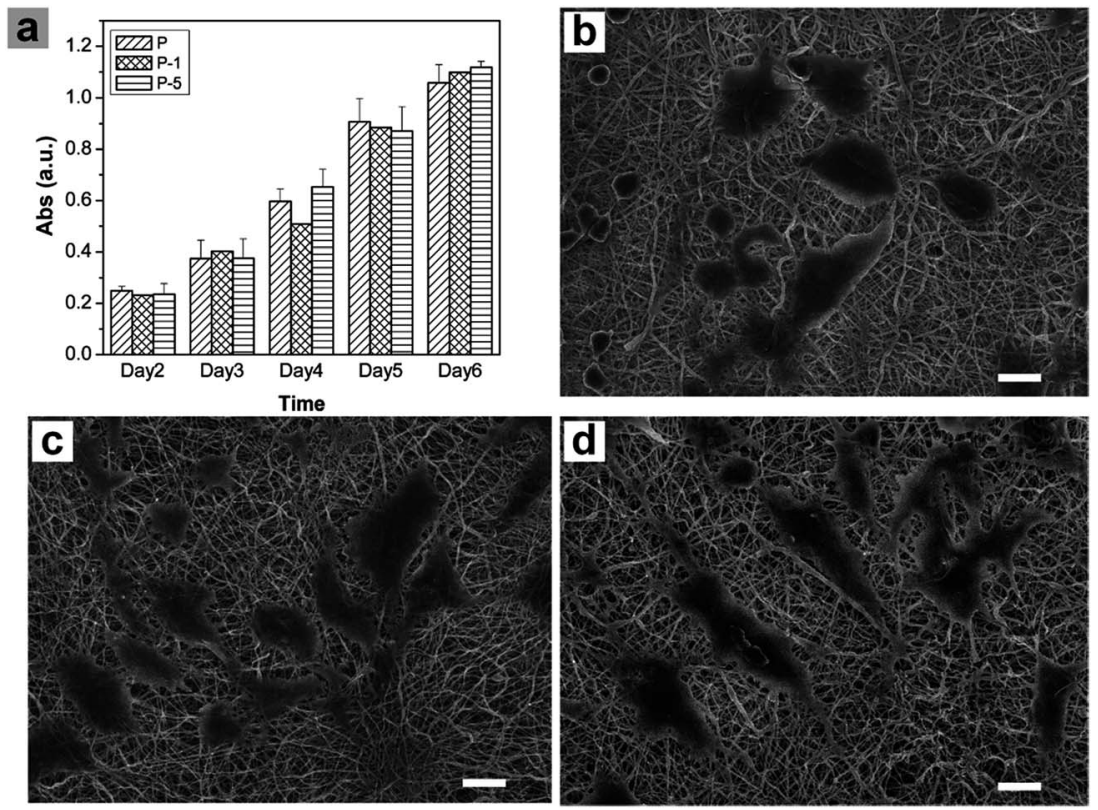

Fig. 6 (a) Monitor of rADSC proliferation on PLGA electrospun membranes using Alamar Blue assay, SEM images of rADSC on (b) P, (c) P-1 and (d) $\mathrm{P}-5$ on day 7. Data $=$ mean $\pm \mathrm{SD} ; n=3$. Scale bars are $20 \mu \mathrm{m}$. 
a

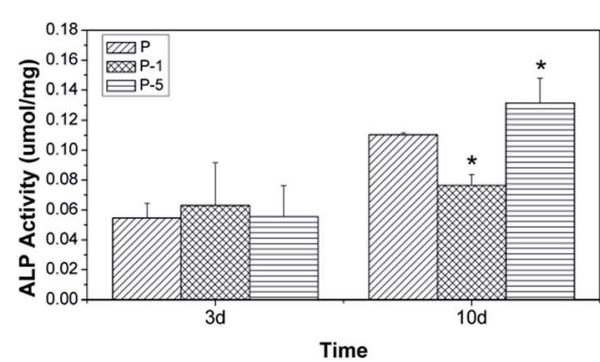

C
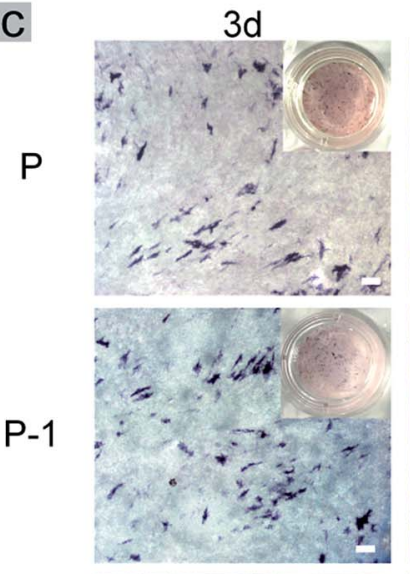

P-5

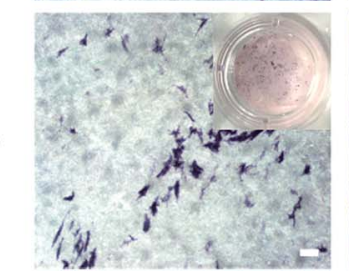

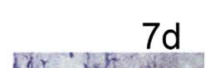
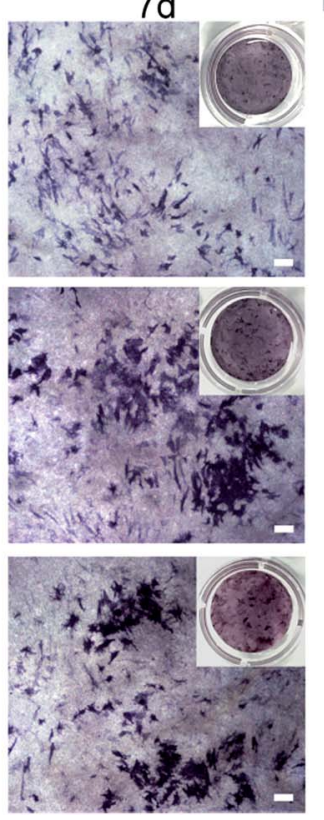

b

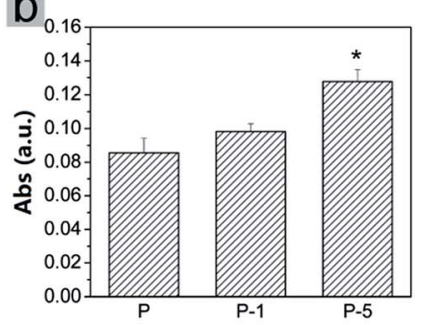

d

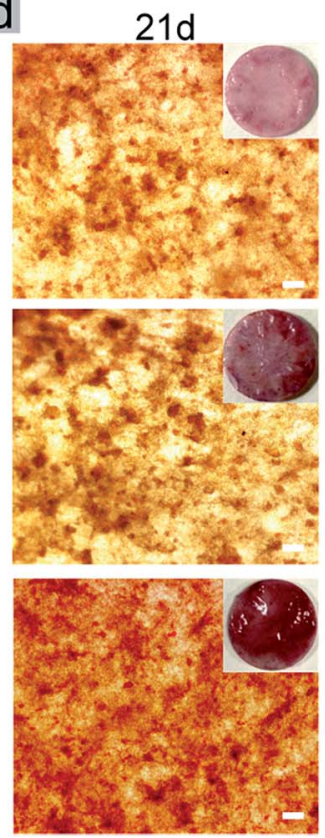

Fig. 7 Investigation of rADSC osteogenic differentiation on PLGA electrospun membranes. (a) Measurement of ALP activity on day 3 and 10, (b) quantification of calcium deposition by dissolving alizarin red stain on day 21, (c) representative light microscope images of rADSC after ALP staining on day 3 and 7, and (d) after Alizarin red staining on day 21. The insets were photos taken with a digital camera. Scale bars represent 100 $\mu \mathrm{m}$. Data was displayed as mean \pm SD for $n=3$. * representing $p<0.05$ when compared with sample $P$.

minerals were hydroxyapatite. All these observations demonstrated that entrapment of PAA-CaP/Zn nanoparticles in PLGA nanofibers enhanced in vitro biomineralization properties of PLGA electrospun composite membranes.

\subsection{In vitro proliferation and osteogenic differentiation of rADSC on PLGA electrospun composite membranes}

Proliferation of rADSC on PLGA electrospun membranes were monitored using Alamar Blue assay. As shown in Fig. 6a, absorbance values which represented cell numbers gradually increased with culturing time, showing that cells proliferated very well on all three samples through 6 days. At each time point, there was no significant difference among these three samples while composite membranes appeared to have more cells. Cell morphology was further observed under SEM. When compared with cells on sample P, cells on P-1 and P-5 were more flattened with plenty of lamellipodia. All these results demonstrated that PLGA electrospun composite membranes offered a favorable microenvironment for rADSC adhesion and proliferation.
The osteogenic differentiation behavior of rADSC on PLGA electrospun membranes was investigated based on monitoring ALP activity and calcium mineral deposition. During osteogenic induction, ALP activity of all samples gradually increased with culturing time (Fig. 7a). In addition, the expression of ALP was confirmed using BCIP/NBT staining. As shown in Fig. 7c, from day 3 to day 7 , the amount of purple stains in microscope images of all samples increased while the color of the digital camera images was darkened. On day 3 , there was no significant difference among these three samples (Fig. 7a and c); when culturing time was elongated to 10 days, the ALP activity of sample P-5 was significantly higher than that of P and P-1 (Fig. 7a). After osteogenic induction for 21 days, calcium mineral deposition was quantified with Alizarin red staining. As shown in Fig. 7b, the absorbance value which represented the calcium mineral deposition increased with the amount of PAA$\mathrm{CaP} / \mathrm{Zn}$ in membranes. For sample P-5, the value was significantly higher than that for sample $\mathrm{P}$ and $\mathrm{P}-1$. Even in the microscope image, the red stains of P-5 were more homogeneous and in digital camera image, the color of P-5 was redder 
than other samples. Based on these data, it can be concluded that PLGA electrospun composite membranes facilitated rADSC osteogenic differentiation, especially sample P-5 which was fabricated with the appropriate amount of PAA-CaP/Zn nanoparticles.

\section{Discussion}

Scaffold is an indispensible component in tissue engineering. To obtain high bioactivity, scaffold was designed to mimic the structure of ECM. Here, electrospinning was selected to fabricate composite membranes as the inter-connecting structure of electrospun fibers is an perfect resemble of nature ECM. ${ }^{6,30}$ To more closely mimic bone ECM which does not only have collagen nanofibers (organic component) but also contains well-dispersed HAp nanoparticles, PAA modified Zn doped HAp-like calcium phosphate (PAA-CaP/Zn) nanoparticles were first fabricated and then further entrapped in PLGA electrospun nanofibers.

Bone HAp is non-stoichiometric at low crystallinity and has other elements in small amounts. Here, the wet chemical reaction was adopted to fabricate HAp nanoparticles ${ }^{31}$ and $\mathrm{Zn}$ was selected for doping to improve the bioactivity. However, the radius of $\mathrm{Zn}^{2+}$ is $0.074 \mathrm{~nm}$, which is slightly smaller than that of $\mathrm{Ca}^{2+}(0.099 \mathrm{~nm}) .{ }^{32}$ Its presence in the reaction system might affect the crystallization process, leading to the formation of amorphous calcium phosphate nanoparticles, corresponding to the broadened XRD peaks (Fig. 1a). In PAA-CaP/Zn, the presence of $\mathrm{Zn}$ was confirmed with EDS. Based on ICP, the atomic ratio of $\mathrm{Zn} /$ Ca was quantified as $5.85 \%$ and the $(\mathrm{Zn}+\mathrm{Ca}) / \mathrm{P}$ atomic ratio was 1.679. Even though the molar ratio of added $(\mathrm{Ca}+\mathrm{Zn}) / \mathrm{P}$ was set as 1.667 , the measured $(\mathrm{Zn}+\mathrm{Ca}) / \mathrm{P}$ atomic ratio was slightly larger. It was highly speculated that there might be some unreacted amorphous $\mathrm{Ca}(\mathrm{OH})_{2}$ molecules adsorbed onto nanoparticles surface since the $\mathrm{pH}$ of reaction system was maintained at $\sim 10$. At such a small doping amount, PAA-CaP/Zn nanoparticles were tested as non-cytotoxic (Fig. 1f). Zn was an indispensible trace element for human health, which was also reported to promote osteocalcin secretion of bone cells, enhance the mineralization of bone tissues and facilitate maturation of bone matrix. ${ }^{32}$ These properties might be beneficial for osteogenic differentiation of rADSC on the fabricated composite membranes.

To improve the dispersity, PAA was used because of its high bonding ability with calcium. The hydrodynamic size of PAA$\mathrm{CaP} / \mathrm{Zn}$ nanoparticles was quantified as $184 \mathrm{~nm}$, which was larger than the reported value. ${ }^{31}$ However, when the same synthetic parameters were followed just without PAA, the hydrodynamic size of the formed particles was quantified as $6817 \mathrm{~nm}$ with zeta potential of $-4.28 \mathrm{mV}$ in mother suspension (pH 10) when reaction was stopped. Even though there might be slight aggregation of $\mathrm{PAA}-\mathrm{Ca} / \mathrm{P}$ nanoparticles (the hydrodynamic size $184 \mathrm{~nm}$ was larger than the size from SEM image), PAA still significantly enhanced the dispersity of calcium phosphate nanoparticles. Based on these characterizations, it can be concluded that well-dispersed PAA-CaP/Zn nanoparticles were successfully fabricated using the simple wet chemical method.
To form composite membranes, PAA-HAp/Zn nanoparticles along with PLGA were dispersed/dissolved in acetone/DMF solvents mixture and then electrospun following the fabrication parameters as described in Section 2.3.1. The diameter of nanofibers was all $\sim 400 \mathrm{~nm}$, which was similar to the size of collagen fiber in ECM. ${ }^{33,34}$ The PAA-HAp/Zn nanoparticles were evenly distributed in PLGA matrix and its presence did not significantly affect the surface morphology of membranes. This was very different from the reported result that PLGA with 5 wt\% HAp electrospun composite membrane had rough nanofibers with HAp aggregates on surface. ${ }^{13}$ The difference was probably because here calcium phosphate nanoparticles were modified with PAA and the dispersity of nanoparticles was significantly improved.

PLGA electrospun composite membranes were designed to repair bone defects. Thus the degradation behavior was investigated. Throughout 28 days of immersion in PBS at $37^{\circ} \mathrm{C}$, fiberlike morphology was all maintained. Among all samples, P-5 was the most stable since surface morphology was nearly not changed (Fig. 4). In long term view, the inclusion of PAA-CaP/Zn nanoparticles might further enhance the repairing effect of PLGA membranes as the degradation by-products of PLGA are acidic while calcium phosphate is basic in nature. It would neutralize these acidic by-products, thus improve the biocompatibility of PLGA membranes. The presence of PAA-HAp/Zn nanoparticles also improved the mechanical properties (Fig. 3) and the in vitro biomineralization properties (Fig. 5) of PLGA membranes. All these results demonstrated the benefits of composite materials which combined the advantages of each component together. This observation was similar to published results. $^{35,36}$

Even though three different ratios of $\mathrm{PAA}-\mathrm{CaP} / \mathrm{Zn}$ nanoparticles were entrapped in PLGA electrospun nanofibers, there was no significant difference regarding surface morphology. Throughout 6 days' proliferation, there was no significant difference among these three samples either. rADSC proliferated well on these membranes for which no surface coating or treatment was applied. It was probably because that the fiber diameter was similar to that of collagen, which resulted in a high specific surface area and hence facilitated cell adhesion. ${ }^{37,38}$ However, the morphology of rADSC was different on these membranes. As shown in Fig. 6, cell spreading area on sample P-1 and P-5 appeared to be larger than that on sample P. This observation was similar to the reported results that cells responded differently to the membranes with different mechanical properties. ${ }^{39,40}$

During osteogenic differentiation, rADSC behaved differently on these membranes (Fig. 7). Sample P-5 demonstrated the strongest enhancing effect on osteogenic differentiation of rADSC. Among all samples, P-5 was the most stable through 28 days of degradation. This stable surface morphology might be beneficial for rADSC differentiation. Meanwhile it was reported that within a certain range, the osteogenic enhancing effect of scaffold material was positively related with its mechanical properties. ${ }^{\mathbf{4 1 , 4 2}}$ Here, P-5 had higher elastic modules. The high amount of PAA-CaP/Zn nanoparticles may also contribute to the enhancing effect on osteogenic differentiation of rADSC as $\mathrm{Zn}^{2+}$ 
has been reported to promote osteocalcin expression of bone cells. ${ }^{32}$ It was highly speculated that all these factors influenced synergistically on osteogenic differentiation of rADSC. Since more stable microenvironment and higher elastic modules were both due to the high amount of PAA-CaP/Zn nanoparticles in P5 , it can be concluded that the designed composition of P-5 facilitated the osteogenic differentiation of rADSC.

\section{Conclusions}

In this paper, well dispersed HAp-like PAA-CaP/Zn nanoparticles were successfully synthesized using a simple precipitation method and these nanoparticles were further entrapped into PLGA electrospun nanofibers. PAA-CaP/Zn nanoparticles were spheroids of $\sim 60 \mathrm{~nm}$. The presence of $\mathrm{Zn}$ significantly lowered the crystallinity and these nanoparticles were noncytotoxic. When included in PLGA nanofibers, these nanoparticles did not compromise the fiber morphology. rADSC proliferated very well on these membranes. The presence of nanoparticles increased mechanical properties and retarded the degradation rate, which together with the appropriate bone ECM mimicking morphology and composition might contribute to the enhancing effect on osteogenic differentiation of rADSC. The fabricated composite membranes demonstrated the potential to be used in bone tissue engineering because of its fiber-like structure and inorganic/organic composition, which needs further in vivo investigations.

\section{Acknowledgements}

This work was supported in part by National Natural Science Foundation of China [grant numbers 51303216, 51103182 and 31270028], Fundamental Research Funds for the Central Universities [grant number 14lgpy12], Guangdong Natural Science Funds for Distinguished Young Scholar [grant number 2016A030306018], Guangdong Provincial Education Department [grant number 2013KJCX005], and the Australia-China Joint Institute for Health Technology and Innovation.

\section{References}

1 D. W. Hutmacher, Biomaterials, 2000, 21, 2529-2543.

2 M. P. Lutolf and J. A. Hubbell, Nat. Biotechnol., 2005, 23, 4755.

3 H. K. Makadia and S. J. Siegel, Polymers, 2011, 3, 1377-1397. 4 K. Rezwan, Q. Z. Chen, J. J. Blaker and A. R. Boccaccini, Biomaterials, 2006, 27, 3413-3431.

5 L. I.-R. Susan, M. G.-K. Genevieve and J. Y. Michael, Biomaterials, 1998, 19, 1405-1412.

6 W.-J. Li, C. T. Laurencin, E. J. Caterson, R. S. Tuan and F. K. Ko, J. Biomed. Mater. Res., 2001, 12, 613-621.

7 B. M. Baker, A. O. Gee, R. B. Metter, A. S. Nathan, R. A. Marklein, J. A. Burdick and R. L. Mauck, Biomaterials, 2008, 29, 2348-2358.

8 S. Gao, M. Calcagni, M. Welti, S. Hemmi, N. Hild, W. J. Stark, G. M. Buergisser, G. A. Wanner, P. Cinelli and J. Buschmann, Injury, 2014, 45, 974-980.
9 A. Haider, K. C. Gupta and I.-K. Kang, BioMed Res. Int., 2014, 2014, 308306.

10 F. Peng, X. Yu and M. Wei, Acta Biomater., 2011, 7, 25852592.

11 M. V. Jose, V. Thomas, K. T. Johnson, D. R. Dean and E. Nyairo, Acta Biomater., 2009, 5, 305-315.

12 M. Li, W. Liu, J. Sun, Y. Xianyu, J. Wang, W. Zhang, W. Zheng, D. Huang, S. Di, Y.-Z. Long and X. Jiang, ACS Appl. Mater. Interfaces, 2013, 5, 5921-5926.

13 L. Lao, Y. Wang, Y. Zhu, Y. Zhang and C. Gao, J. Mater. Sci.: Mater. Med., 2011, 22, 1873-1884.

14 D. Li, H. Sun, L. Jiang, K. Zhang, W. Liu, Y. Zhu, J. Fangteng, C. Shi, L. Zhao, H. Sun and B. Yang, ACS Appl. Mater. Interfaces, 2014, 6, 9402-9410.

15 N. Hild, O. D. Schneider, D. Mohn, N. A. Luechinger, F. M. Koehler, S. Hofmann, J. R. Vetsch, B. W. Thimm, R. Müller and W. J. Stark, Nanoscale, 2011, 3, 401-409.

16 H. B. John and A. Alison, Nutr. Res. Rev., 1992, 5, 167-188.

17 W. Xue, K. Dahlquist, A. Banerjee, A. Bandyopadhyay and S. Bose, J. Mater. Sci.: Mater. Med., 2008, 19, 2669-2677.

18 M. Hie and I. Tsukamoto, Eur. J. Pharmacol., 2011, 668, 140146.

19 A. Wang, H. Yin, D. Liu, H. Wu, M. Ren, T. Jiang, X. Cheng and Y. Xu, Mater. Lett., 2007, 61, 2084-2088.

20 S. Pujari-Palmer, S. Chen, S. Rubino, H. Weng, W. Xia, H. Engqvist, L. Tang and M. K. Ott, Biomaterials, 2016, 90, 1-11.

21 E. Arrigoni, S. Lopa, L. De Girolamo, D. Stanco and A. T. Brini, Cell Tissue Res., 2009, 338, 401-411.

22 Y. Ramaswamy, C. Wu, A. V. Hummel, V. Combes, G. Grau and H. Zreiqat, Biomaterials, 2008, 28, 4392-4402.

23 H. Valiense, M. Barreto, R. F. Resende, A. T. Alves, A. M. Rossi, E. Mavropoulos, J. M. Granjeiro and M. D. Calasans-Maia, J. Biomed. Mater. Res., Part B, 2016, 104, 274-282.

24 T. Kokubo and H. Takadama, Biomaterials, 2006, 27, 29072915.

25 K. Kato, Y. Eika and Y. Ikada, J. Mater. Sci., 1997, 32, 55335543.

26 S.-C. Liou, S.-Y. Chen and D.-M. Liu, Biomaterials, 2003, 24, 3981-3988.

27 R. Gonzalez-McQuire, J.-Y. Chane-Ching, E. Vignaud, A. Lebugle and S. Mann, J. Mater. Chem., 2004, 14, 2277.

28 R. N. Panda, M. F. Hsieh and R. J. Chung, J. Phys. Chem. Solids, 2003, 64, 193-199.

29 Z. Cao, D. Wang, L. Lv, Y. Gong and Y. Li, RSC Adv., 2016, 6, 10641-10649.

30 Z.-M. Huang, Y. Z. Zhang, M. Kotaki and S. Ramakrishna, Compos. Sci. Technol., 2003, 63, 2223-2253.

31 Q. Wang, Z. Gu, S. Jamal, M. S. Detamore and C. Berkland, Tissue Eng., Part A, 2013, 19, 2586-2593.

32 E. S. Thian, T. Konishi, Y. Kawanobe, P. N. Lim, C. Choong, B. Ho and M. Aizawa, J. Mater. Sci.: Mater. Med., 2013, 24, 437-445.

33 L. A. Smith and P. X. Ma, Colloids Surf., B, 2004, 39, 125-131. 34 E. Tom and B. Jonathan, J. Cell Biol., 1972, 54, 626-637. 
35 Y. Ito, H. Hasuda, M. Kamitakahara, C. Ohtsuki, M. Tanihara, I. K. Kang and O. H. Kwon, J. Biosci. Bioeng., 2005, 100, 43-49.

36 H. W. Kim, H. H. Lee and J. C. Knowles, J. Biomed. Mater. Res., Part A, 2006, 79, 643-649.

37 M. R. Williamson, R. Black and C. Kielty, Biomaterials, 2006, 27, 3608-3616.

38 J. Stitzel, J. Liu, S. J. Lee, M. Komura, J. Berry, S. Soker, G. Lim, M. Van Dyke, R. Czerw, J. J. Yoo and A. Atala, Biomaterials, 2006, 27, 1088-1094.
39 S. Ouasti, R. Donno, F. Cellesi, M. J. Sherratt, G. Terenghi and N. Tirelli, Biomaterials, 2011, 32, 6456-6470.

40 V. E. G. Diederich, P. Studer and A. Kern, Biotechnol. Bioeng., 2013, 110, 1508-1519.

41 B. Jankovic, J. Pelipenko, M. Skarabot, I. Musevic and J. Kristl, Int. J. Pharm., 2013, 455, 338-347.

42 J. H. Lee, H. K. Park and K. S. Kim, Biochem. Biophys. Res. Commun., 2016, 473, 752-757. 